Original research article

\title{
A dynamic cell recruitment process drives growth of the Drosophila wing by overscaling the vestigial expression pattern
}

\author{
Luis Manuel Muñoz-Nava ${ }^{\text {a }}$, Hugo Ariel Alvarez ${ }^{\text {b,c }}$, Marycruz Flores-Flores ${ }^{\text {a }}$, Osvaldo Chara ${ }^{\text {b,d, }}$ \\ Marcos Nahmad ${ }^{\mathrm{a}, *}$ \\ ${ }^{a}$ Department of Physiology, Biophysics, and Neurosciences, Center for Research and Advanced Studies of the National Polytechnical Institute (Cinvestav-IPN), Mexico City, \\ 07360 , Mexico \\ ${ }^{\mathrm{b}}$ Systems Biology Group (SysBio), Institute of Physics of Liquids and Biological Systems (IFLYSIB), National Scientific and Technical Research Council (CONICET) and \\ University of La Plata (UNLP), La Plata, B1900BTE, Argentina \\ ${ }^{\mathrm{c}}$ Department of Biological Sciences, Faculty of Exact Sciences, University of La Plata (UNLP), La Plata, 1900, Buenos Aires, Argentina \\ ${ }^{\mathrm{d}}$ Center for Information Services and High Performance Computing (ZIH), Technische Universität Dresden (TUD), Dresden, 01069, Germany
}

\section{A R T I C L E I N F O}

\section{Keywords:}

Organ growth

Patterning

Vestigial

Cell recruitment

Scaling

Drosophila wing disc

\begin{abstract}
A B S T R A C T
Organs mainly attain their size by cell growth and proliferation, but sometimes also grow through recruitment of undifferentiated cells. Here we investigate the participation of cell recruitment in establishing the pattern of Vestigial (Vg), the product of the wing selector gene in Drosophila. We find that the Vg pattern overscales along the dorsal-ventral (DV) axis of the wing imaginal disc, i.e., it expands faster than the DV length of the pouch. The overscaling of the $\mathrm{Vg}$ pattern cannot be explained by differential proliferation, apoptosis, or oriented-cell divisions, but can be recapitulated by a mathematical model that explicitly considers cell recruitment. When impairing cell recruitment genetically, we find that the Vg pattern almost perfectly scales and adult wings are approximately $20 \%$ smaller. Conversely, impairing cell proliferation results in very small wings, suggesting that cell recruitment and cell proliferation additively contribute to organ growth in this system. Furthermore, using fluorescent reporter tools, we provide direct evidence that cell recruitment is initiated between early and mid third-instar larval development. Altogether, our work quantitatively shows when, how, and by how much cell recruitment shapes the $\mathrm{Vg}$ pattern and drives growth of the Drosophila wing.
\end{abstract}

\section{Introduction}

Organ growth during development is orchestrated by morphogens, which are signaling molecules that determine gene expression in a noncell autonomous manner and also act as mitogens (Day and Lawrence, 2000; Schwank and Basler, 2010; Dekanty and Milán, 2011; Lander, 2011; Wartlick et al., 2011; Bryant and Gardiner, 2016; Vollmer et al., 2017; Gou et al., 2020). However, growth may also be driven independently of morphogen-induced cell proliferation, by a growth-by-patterning mechanism in which a differentiation pattern expands at the expense of the incorporation of undifferentiated cells. This mechanism, also known as inductive assimilation or cell recruitment, has been identified in both vertebrate and invertebrate development such as in the eye (Heberlein et al., 1995; Strutt et al., 1995) and wing (Baena-López and García-Bellido, 2003; Zecca and Struhl, 2007a) of the fruit fly, Drosophila melanogaster, as well as in the mammalian thyroid
(Fagman et al., 2006) and kidney (Lindström et al., 2018). Little is known, however, about the dynamic properties of patterning by cell recruitment and how much organ growth can be gained through cell recruitment relative to cell growth and proliferation. These questions are generally difficult to address during normal development because the dynamics of patterning and cell proliferation occur simultaneously and it is challenging to isolate the contribution of each of these mechanisms to organ size. The Drosophila wing imaginal disc is a useful system to tackle this problem since previous studies have partly identified the molecular players of recruitment (Zecca and Struhl, 2007a, 2010) and genetic tools allow manipulation and quantitative assessment of patterning and growth (Hariharan and Bilder, 2006; Beira and Paro, 2016).

In Drosophila, wing fate is determined by the expression of the wing selector gene, vestigial $(\mathrm{vg})$, in the pouch of the wing imaginal disc (Williams et al., 1991). The absence of $v g$ results in loss of wing blade identity (Williams et al., 1991; Williams et al., 1993), whereas ectopic

\footnotetext{
* Corresponding author.

E-mail address: mnahmad@fisio.cinvestav.mx (M. Nahmad).
} 
expression of $v g$ in other imaginal discs could induce transformation into wing-like tissue (Williams et al., 1994; Kim et al., 1996; Halder et al., 1998; Baena-López and García-Bellido, 2003). Therefore, adult wing size depends on the size and shape of the $\mathrm{Vg}$ pattern established during the larval stage.

$v g$ expression is controlled at least by two enhancers, the $v g$ boundary enhancer $\left(v g^{B E}\right)$ that responds to Notch signaling and results in high expression of Vg at the DV boundary (Irvine and Vogt, 1997), and the $v g$ quadrant enhancer $\left(v g^{Q E}\right)$ that is activated by Wingless $(\mathrm{Wg})$ and Decapentaplegic (Dpp) signaling (Kim et al., 1996; Zecca and Struhl, 2007b; Klein and Martínez-Arias, 1998). Along the DV axis, the combined activation of the $v g^{B E}$ and $v g^{Q E}$ results in a gradient of $\mathrm{Vg}$ that peaks at the DV boundary (Baena-López and García-Bellido, 2006). As the disc grows, the lineage of cells abutting the DV boundary maintains its transcriptional expression of $v g$ through Polycomb/Trithorax Responsive Elements and Vg itself (Klein and Martínez-Arias, 1999; Pérez et al., 2011; Ahmad et al., 2019). In addition, the $v g^{Q E}$ is the responsive element of a cell recruitment process that depends on a feed-forward signal sent by Vg-expressing cells to non-expressing cells (Zecca and Struhl, 2007a). In 2010, Zecca and Struhl identified the following molecular details of the recruitment process (Zecca and Struhl, 2010): (1) Vg-expressing cells transcriptionally repress the protocadherin-encoding gene dachsous ( $d s)$, resulting in complementary patterns of $\mathrm{Vg}$ and Ds expression; (2) the boundary of the $\mathrm{Vg}$ /Ds expression domains facilitates Fat-Ds polarization, and (3) the polarization signaling induces nuclear shuttling of Yorkie (Yki), the transcriptional factor downstream of the Warts-Hippo tumor-suppressor pathway (Yu et al., 2015), where it promotes $v g$ expression transcriptionally. This process results in a new Vg-expressing cell, thus propagating the $\mathrm{Vg}$ pattern. As new cells are recruited into the Vg domain, the Ds pattern is pushed outwards radially by Vg-dependent transcriptional repression from the center of the wing pouch.

While previous studies have provided experimental evidence of the recruitment process by showing that Vg-expressing mosaics can induce expression of a $v g^{Q E}$ reporter non-cell-autonomously (Zecca and Struhl, 2007a, 2010), the contribution of cell recruitment to wild-type patterning and growth of the wing disc has not yet been investigated. Here, we precisely address this question by quantitatively examining the spatiotemporal formation of the $\mathrm{Vg}$ gradient and asking if these dynamics can be explained by a cell recruitment mechanism. We show that cell recruitment affects the shape of the $\mathrm{Vg}$ pattern and contributes to final size of the adult Drosophila wing.

\section{Results}

\subsection{The Vg pattern overscales with respect to disc size along the DV axis}

In order to investigate how the $\mathrm{Vg}$ pattern changes as a function of tissue size, we quantified $\mathrm{Vg}$ expression in wild-type wing discs during the third larval instar $\left(85-120 \mathrm{~h}\right.$ After Egg Laying (AEL) at $25^{\circ} \mathrm{C}$ ) as a function of DV position in a central region of the pouch delimited by the dorsal and ventral epithelial folds (Fig. 1A; Sup. Fig. 1; Sup. Text). These folds robustly appear in the disc at about $85 \mathrm{~h} \mathrm{AEL}$ at $25{ }^{\circ} \mathrm{C}$ (Sui et al., 2012) and serve as references to define the length of the DV axis within the pouch area (Fig. 1A). We found that DV length correlates with disc age (Fig. 1B), and therefore, we used DV length as an indirect measure of developmental time to investigate the spatiotemporal dynamics of the $\mathrm{Vg}$ pattern. Fig. 1C-E shows three sample discs of different DV lengths stained for $\mathrm{Vg}$ and DAPI. Since fluorescence levels drop close to the folds due to tissue geometry (Fig. 1C' - E'), we used normalized DAPI levels to correct Vg expression (see Sup. Text). After DAPI correction, Vg is expressed in a concentration gradient in all the discs examined, with maximum levels near the DV border and decreasing towards the ventral and dorsal folds (Fig. 1C"',- E"'). However, when we plotted the normalized Vg patterns of all discs in relative units (i.e., \% of DV length; Fig. 1F), we observed some dynamic features of the Vg patterns. First, we noticed that the relative width of the $\mathrm{Vg}$ gradient (width to DV length ratio) on average increases when plotted vs. DV length (Fig. 1G, Sup. Fig. 2). This result indicates that the Vg gradient overscales with respect to disc size, i.e., that the $\mathrm{Vg}$ pattern expands further than what would be expected by uniform growth of the disc along the DV axis. Second, Vg levels significantly increase in cells located near the dorsal and ventral folds throughout the third instar (Fig. 1H). Finally, we noticed a ventral shift of the relative position of the Vg maximum (Sup. Fig. 3A); this shift suggests that the dorsal compartment grows faster than the ventral compartment. In summary, we found that during normal development, the scale, shape, and symmetry of the Vg gradient changes with respect to disc size along the DV axis.

\subsection{The dynamics of the Vg pattern is not a result of differential cell proliferation or apoptosis}

We considered if overscaling of the Vg pattern could be explained by differences in cell proliferation or apoptosis between Vg-expressing $v s$. non-Vg expressing cells within the pouch. For example, cells near the periphery of the pouch may be experiencing higher apoptosis rates or $\mathrm{Vg}$ expressing cells may proliferate faster than cells outside of the $\mathrm{Vg}$ domain, explaining the overscaling of the $\mathrm{Vg}$ pattern. To test these possibilities, we first examined the expression of the pro-apoptotic marker Caspase 3 (Cas3) throughout development and found that apoptosis occurs at very low frequency in the wing pouch (Sup. Fig. 4); this is consistent with a previous study (Milán et al., 1997). Second, we examined cell proliferation within the pouch and found that it is approximately uniform during most of the third instar, except for cells abutting the DV boundary that almost do not proliferate, in agreement with previous studies (Sup. Fig. 5A and B; Schwank et al., 2011; Wartlick et al., 2011; Mao et al., 2013). We conclude that the overscaling of the $\mathrm{Vg}$ pattern cannot be accounted by differences in cell proliferation or apoptosis between cells within and outside the Vg domain.

\subsection{Mathematical modeling of cell recruitment predicts the overscaling of the Vg pattern}

In order to investigate if the dynamics of the $\mathrm{Vg}$ gradient could be explained by a cell recruitment mechanism, we modeled the distribution of $\mathrm{Vg}$ in the wing pouch by means of a multi-scale model (see Sup. Text for a full description of the model). The model combines an ordinary differential equation to account for the rate of change of $\mathrm{Vg}$ concentration in each cell $\left(V g^{i}\right)$ with a 2D Cellular Potts Model (CPM; Graner and Glazier, 1992; Glazier and Graner, 1993) describing the cellular dynamics (Fig. 2A and Sup. Fig. 6A and B). We assumed that cells produce $\mathrm{Vg}$ by two mechanisms, while there is only one sink modeled as a linear degradation. The first production term assumes that $\mathrm{Vg}$ expression responds to the concentration of a given non-scaling morphogen M (Fig. 2A and Sup. Text). The second cellular source of $\mathrm{Vg}$ is our quantitative formulation of the recruitment mechanism. In particular, we proposed that this production term depends on the difference in $\mathrm{Vg}$ concentration between the actual cell and the average of the concentration of its first neighbors by a second order Hill function (Fig. 2A and Sup. Fig. 6C). Hence, this production term becomes relevant when the concentration of the cell is different from the average of its neighbors and negligible when they are similar. As a control, we also considered a model without the recruitment term. In order to compare the simulations of our mathematical model with our experimental data, we subdivided the discs into 4 groups according to their DV length (Sup. Fig. 7A) and obtained the average $\mathrm{Vg}$ profile of the discs in each group (Sup. Fig. 7B). To account for disc growth, we explicitly included homogeneous cell proliferation in which the cell cycle parameters were fitted to the average number of cells in each of these four groups (Sup. Fig. 8 and Sup. Text). Schematics of the simulated Vg expression patterns corresponding to each of the groups are shown in Fig. 2B-E (without cell recruitment) and Fig. 2B'-E' (with cell recruitment).

We examined whether the models (with and without the recruitment 

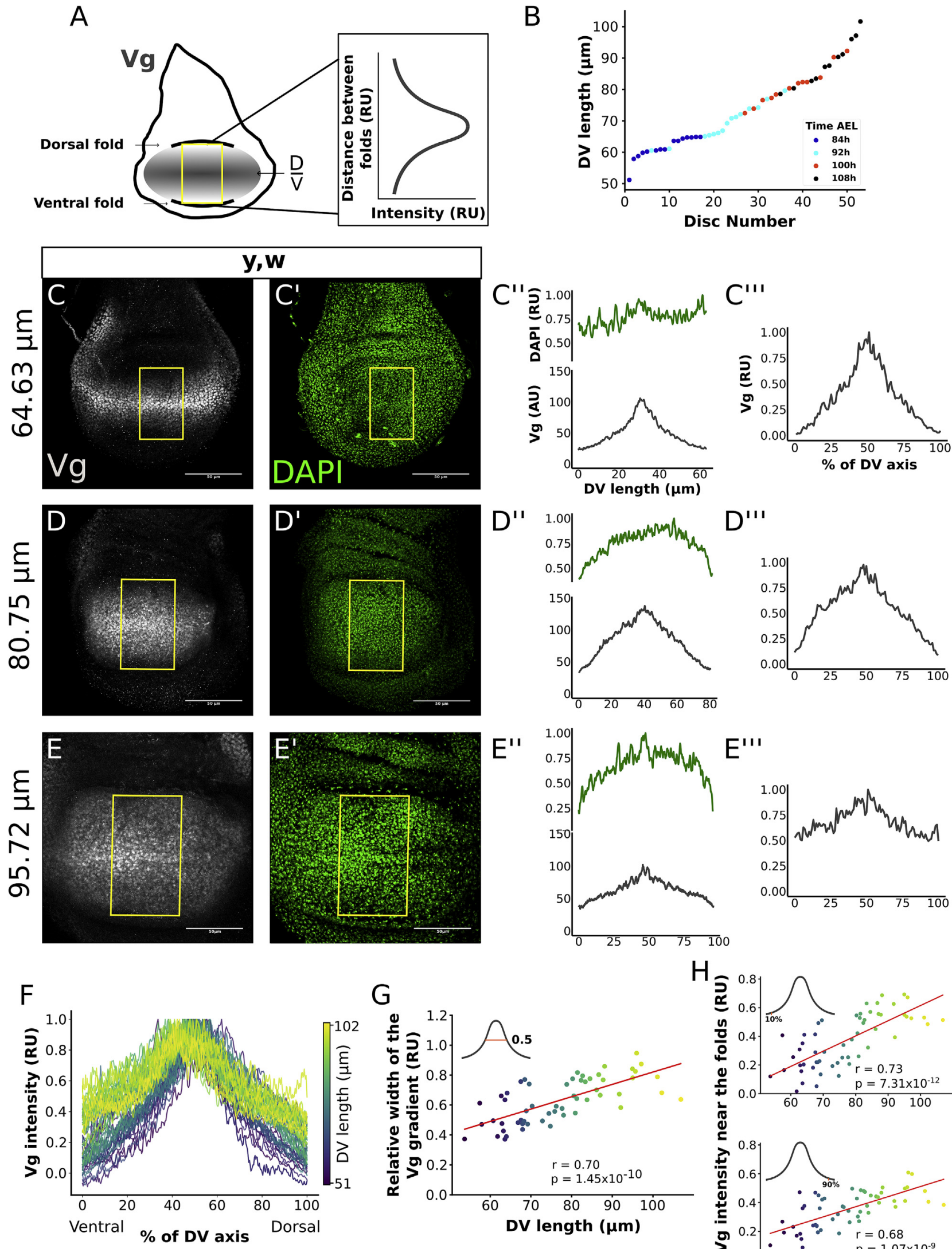

G
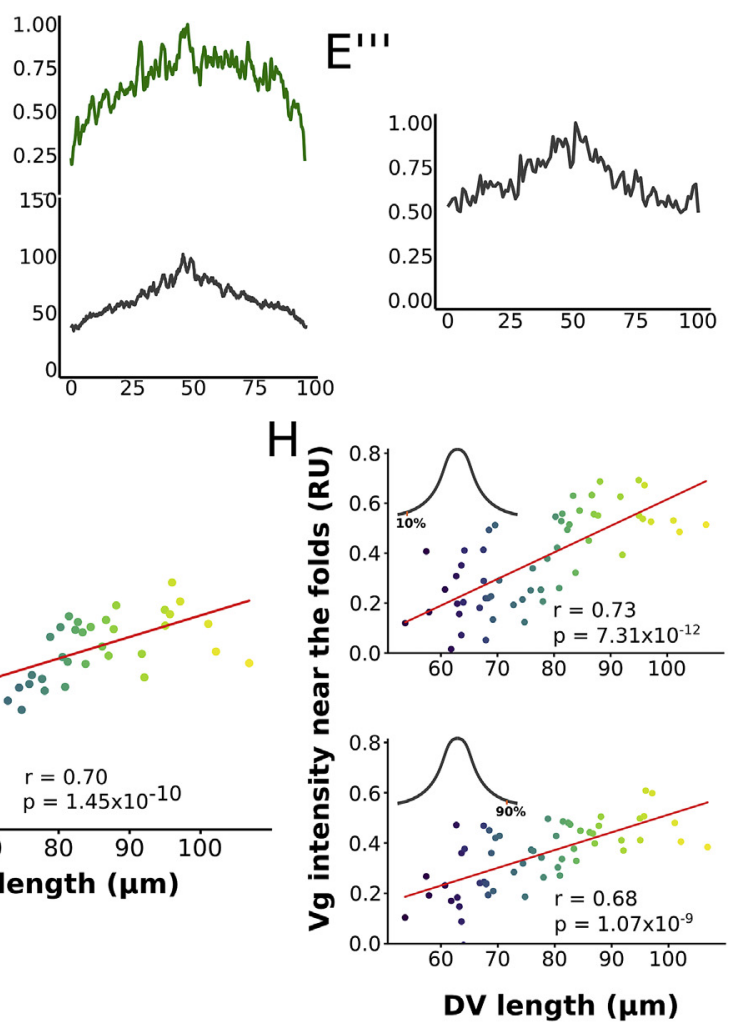

(caption on next page) 


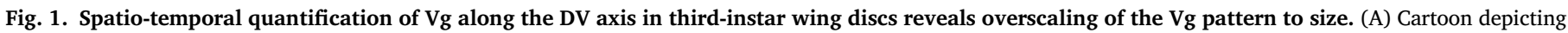

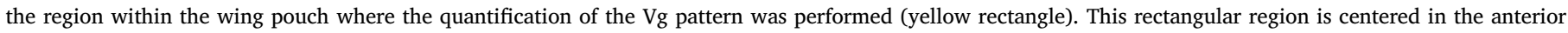

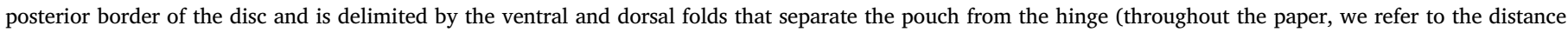

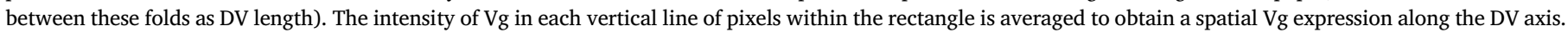

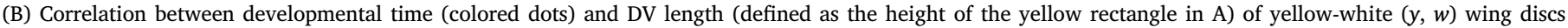

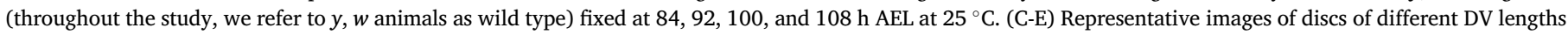

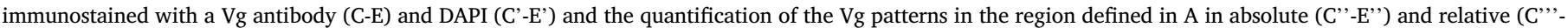

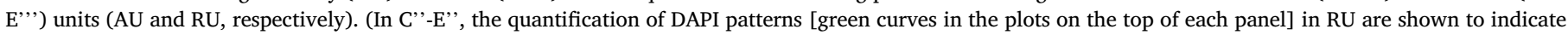

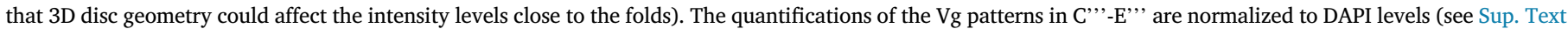

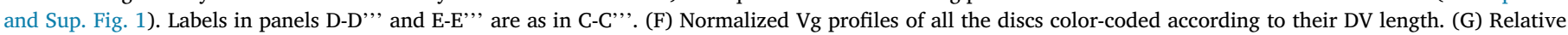

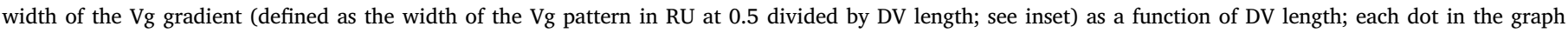

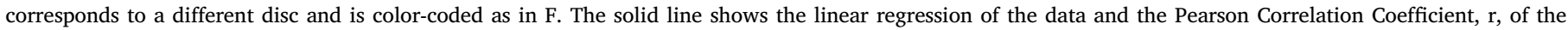

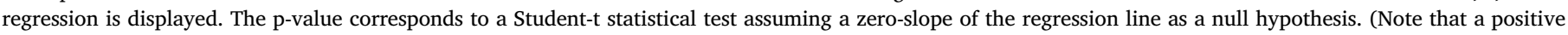

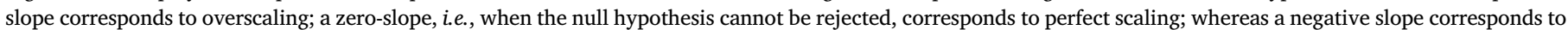

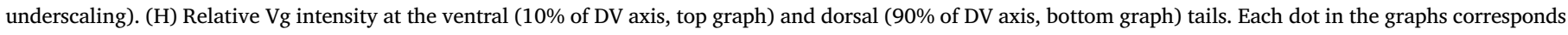
to a different disc and is color-coded as in F. Linear regression and statistical analysis is performed as in G.

term) could explain the spatiotemporal expansion of Vg shown in Fig. 1 by a fitting procedure where we varied the parameter values of each of the models and minimized a target function of the residuals between experimental and simulated Vg expression data. (For simplicity, we did not take into account differences between dorsal and ventral compartments [Sup. Fig. 3], so we only compared experimental and simulated data in the dorsal compartment; Sup. Fig. 9). The best-fit simulation of the model with the recruitment term reasonably recapitulates the experimental overscaling in each of the four groups (Fig. 2B"'-E', blue line vs. black line; Sup. Fig. 10; Sup. Video 1; Sup. Text) and the minimum of the target function is clearly identified within the parameter space (Sup. Fig. 11A-D, blue dots, minimum marked with a green circle). In contrast, the best-fit solution of the model without the recruitment term does not reproduce the experimental data (Fig. 2B"'-E', red line $v s$. black line; Sup. Video 1).

Supplementary video related to this article can be found at https:// doi.org/10.1016/j.ydbio.2020.03.009.

Finally, since cell divisions in the wing pouch are oriented along the proximal-distal axis (González-Gaitán et al., 1994; Resino et al., 2002; Baena-López et al., 2005; Mao et al., 2013), we used our mathematical model to ask whether the overscaling effect could be enhanced by assuming that cell divisions are oriented radially. We found that essentially the same fit was achieved in a model that assumes radially-oriented cell divisions $v s$. a model where cell divisions are randomly oriented (Sup. Fig. 12). We conclude that a mathematical model encoding a mechanism of cell recruitment may explain the overscaling dynamics of the $\mathrm{Vg}$ gradient regardless of the orientation of cell divisions.

\subsection{Blocking Vg expression in ds-expressing cells disrupts the overscaling of the Vg gradient and nearly results in perfect scaling}

To verify the predictions of our mathematical model, we expressed an interference RNA for $v g\left(v g^{R N A i}\right)$ under $d s$ control $\left(d s>v g^{R N A i}\right)$ using the Drosophila Gal4-UAS system (Brand and Perrimon, 1993; Sup. Fig. 13). Since cell recruitment works by expanding $\mathrm{Vg}$ expression at the expense of $d s$-expressing cells (Zecca and Struhl, 2010), we expect cell recruitment will be blocked in $d s>v g^{R N A i}$ discs. Moreover, if cell recruitment is indeed responsible for the overscaling of the $\mathrm{Vg}$ gradient in vivo, we predict that the overscaling phenotype will be lost in $d s>v g^{R N A i}$ discs. We quantified the patterns of $\mathrm{Vg}$ as we did for the wild-type discs (Fig. 3A-C). As predicted, on average the overscaling of the $\mathrm{Vg}$ pattern is mostly lost (Fig. 3D, compare to Fig. 1F). Particularly, when we plotted the relative width of the $\mathrm{Vg}$ pattern and the $\mathrm{Vg}$ intensity near the folds $v s$. DV length in these discs, we found that there is still a slight overscaling phenotype, although this is much more subtle than in wild-type discs (Fig. 3E and F; compare with Fig. $1 \mathrm{G}$ and H).

We considered if the loss of $\mathrm{Vg}$ overscaling in $d s>v g^{R N A i}$ discs could result from alternative mechanisms. For example, $d s>v g^{R N A i}$ discs may experience higher cell proliferation or lower apoptosis rates at the edges of the pouch due to cell competition or changes in the slope of the gradient (Baena-López and García-Bellido, 2006). However, we found that the distribution of cell proliferation and apoptosis are very similar in control and $d s>v g^{R N A i}$ discs (Sup. Fig. 5). Next, we examined experimentally if the expansion of the $\mathrm{Vg}$ pattern is the result of oriented cell divisions. Since $\mathrm{Vg}$ inhibits $d s$ expression transcriptionally (Zecca and Struhl, 2007a) and the Fat-Ds pathway dictates the orientation of cell divisions along the proximal-distal direction (Baena-López et al., 2005), it is possible that the orientation of cell divisions could be affected in $d s>v g^{R N A i}$ discs, resulting in a narrower Vg gradient. We did not see significant differences in the patterns of cell divisions in $d s>$ $v g^{R N A i}$ with respect to control discs (Sup. Fig. 14). Finally, we also examined whether changes in cell sizes under this genetic perturbation could explain the changes in the dynamics of the $\mathrm{Vg}$ gradient. Once again, we did not appreciate significant differences in cell sizes between control and $d s>v g^{R N A i}$ discs (Sup. Fig. 15). We conclude that the dynamics of the $\mathrm{Vg}$ pattern in wild type and $d s>v g^{R N A i}$ discs cannot be attributed to changes in cell proliferation, apoptosis, orientation of cell divisions, or cell size.

\subsection{A time-dependent cell recruitment process contributes to the Vg pattern during normal development}

So far, our evidence of a cell recruitment mechanism that patterns the $\mathrm{Vg}$ gradient has been indirect. In order to provide direct evidence that cell recruitment does occur during normal development, we used a recent tool of dual-color fluorescent reporters, known as TransTimer (He et al., 2019), to examine the dynamics of the $v g^{Q E}$. The TransTimer expresses a nuclear GFP more rapidly than a nuclear RFP under the Gal4-UAS system. We quantified the reporters of the TransTimer under the control of $v g^{Q E} \mathrm{Gal} 4$ in fixed discs of different DV lengths (Fig. 4). In this experiment, cells that activated the $v g^{Q E}$ within the last 2-3 h will turn on GFP but no RFP (more precisely, they will have a RFP to GFP ratio below a threshold). We will refer to these cells as "green cells" (see Fig. 4A). In contrast, cells that have been activating the $v g^{Q E}$ for longer will express both GFP and RFP (i.e. have a RFP to GFP ratio above the threshold). We found several green cells in early-to-mid third-instar wing discs but little or no green cells in older/larger discs (Fig. 4B-E). Green cells are mostly located in the periphery of the $v g^{Q E}$ pattern, either adjacent to the $v g^{B E}$ domain (likely, these are cells that just transition $v g$ expression from the $v g^{B E}$ to the $v g^{Q E}$; Fig. 4B", arrowheads), or at the edges of the wing pouch (Fig. 4B"' arrows). Moreover, we found that some green cells are located up to 16 cells away from the DV boundary (asterisk in Fig. 4B-B"' magnification), which is beyond the reported range of $\mathrm{Wg}$ signaling (Chaudhary et al., 2019; see Discussion). Taken together, this experiment shows that new cells are incorporated into the $v g^{Q E}$ pattern, providing direct evidence of cell recruitment initiation during early-to-mid third instar development. 


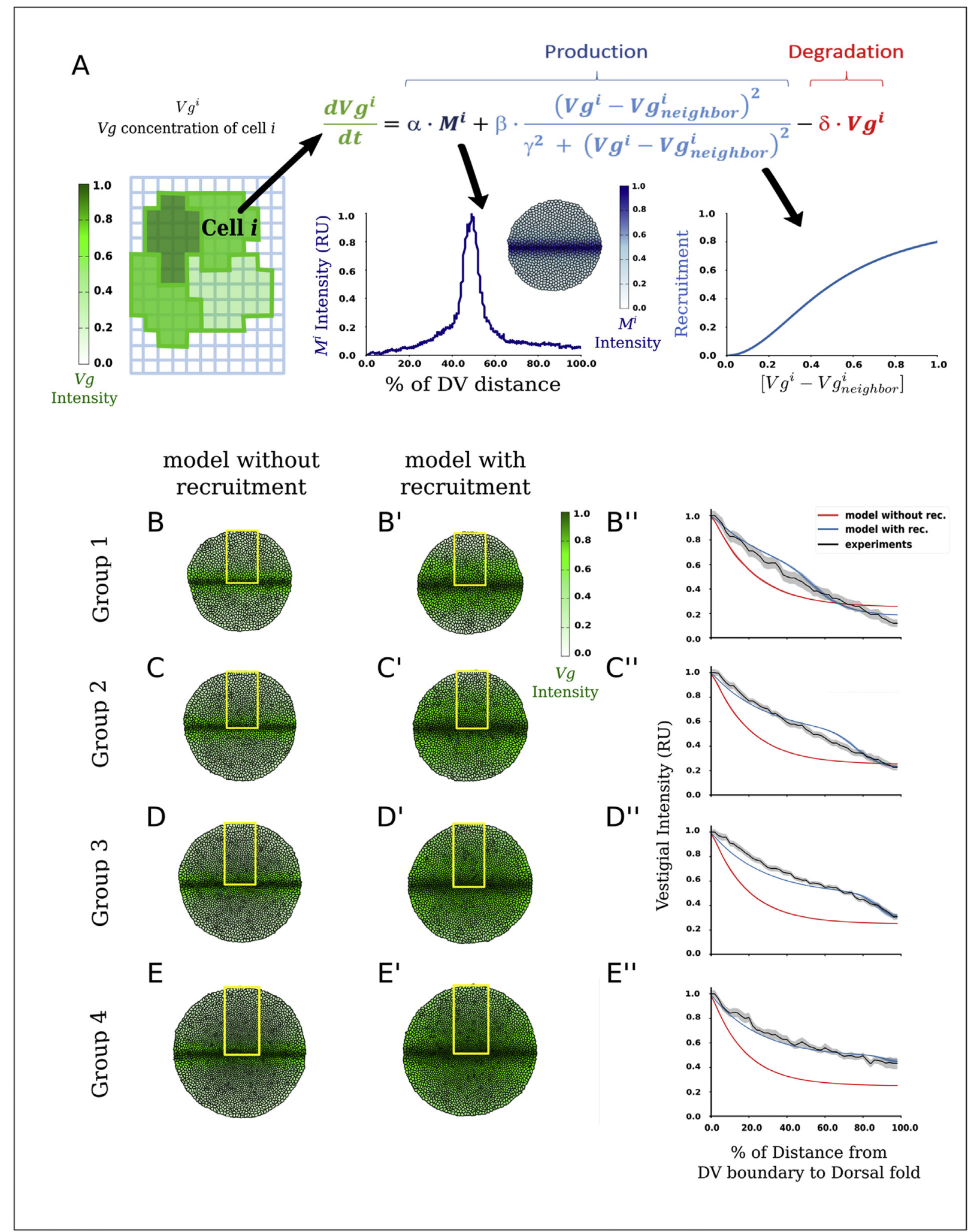

Fig. 2. Multi-scale mathematical modeling with recruitment recapitulates Vg overscaling. (A) Outline of the model (see Sup. Text for further details). The dynamics of $\mathrm{Vg}$ concentration in each cell is given by an ordinary differential equation including: a spatial-dependent production (first term) that models the concentration-dependent effect of source signal or morphogen M, our formulation of the recruitment mechanism (second term) that depends on a Hill function of the difference in Vg concentration between the actual cell and the average of its neighbors, and linear degradation (third term). As a control, we considered a model without the recruitment term. (B-E) Simulated tissues showing Vg profiles of the model without the recruitment term (B-E), and with the recruitment term (B'-E') at four different simulated times corresponding to the average pouch areas of discs in Groups 1-4 (see Sup. Figs. 7A and 8). (B'-E') Simulated patterns of Vg in the dorsal compartment (obtained by horizontally averaging the simulated Vg patterns within the yellow rectangles in B-E and B'-E') from the model with (blue curves) or without (red curves) the recruitment term, using parameter values that best fit the average experimental Vg profiles (black curves; shades represent the Standard Error of the Mean of all the experimental profiles in each group; Sup. Fig. 7B). 

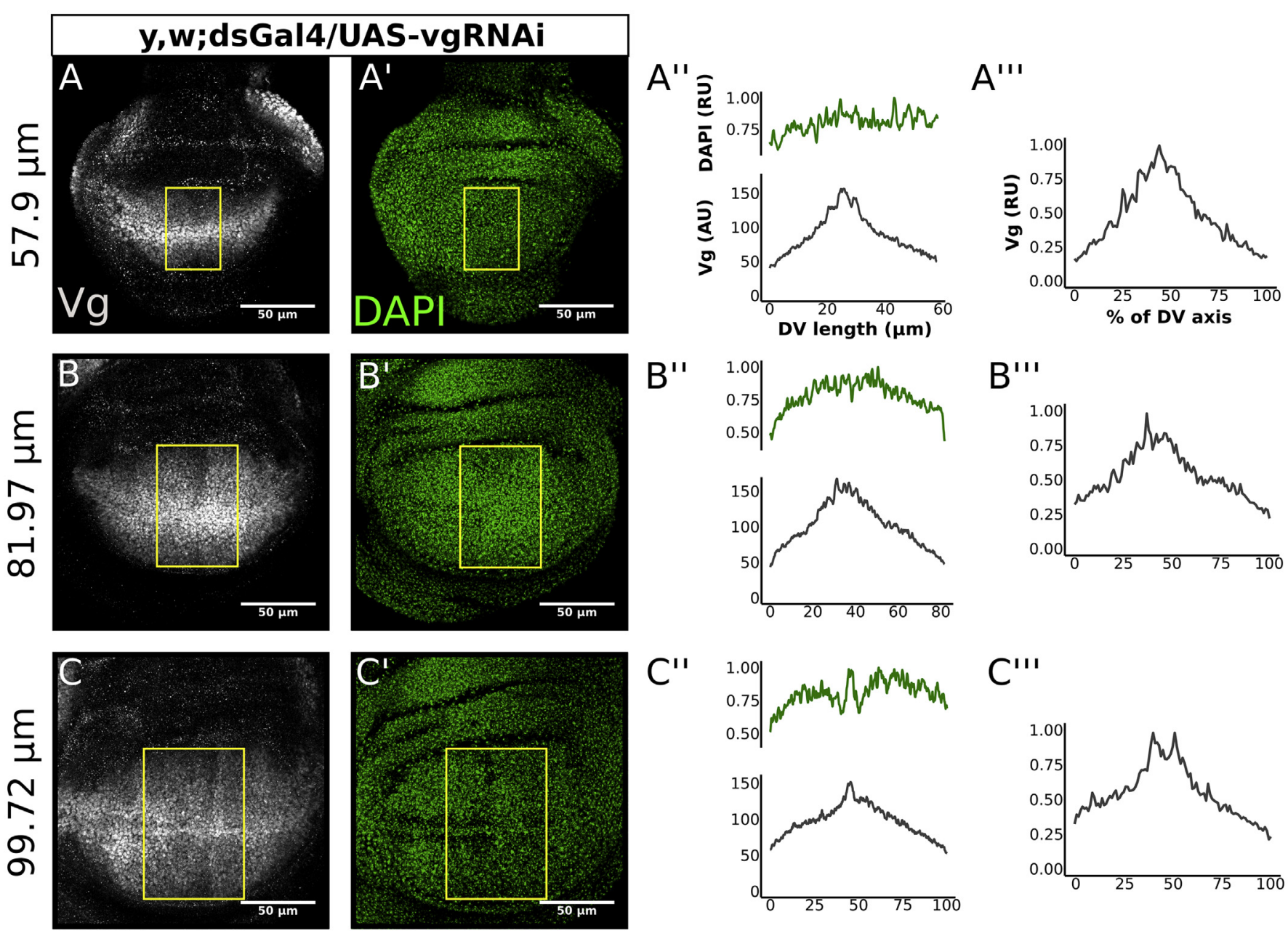

$B^{\prime \prime \prime}$

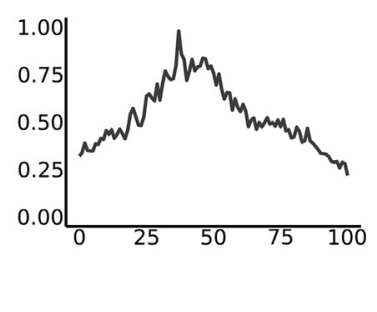

$C^{\prime \prime \prime}$

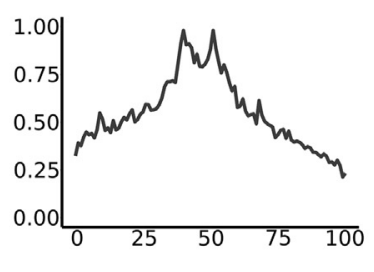

$\mathrm{F}$

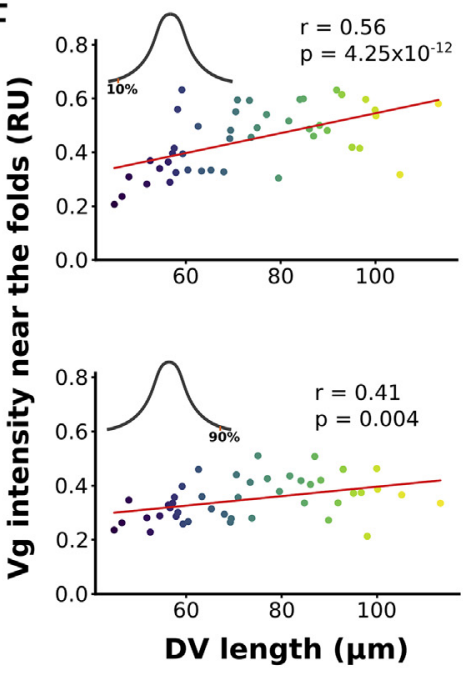

Fig. 3. Genetic impairment of cell recruitment by $d s>v g^{R N A i}$ results in almost perfect scaling of the $\mathrm{Vg}$ pattern. (A-C) Representative images of third-instar wing discs in which cell recruitment was blocked by expressing $v g^{R N A i}$ in the domain of $d s$ using the Gal4-UAS system. Discs were immunostained with a Vg antibody (A-C) and DAPI (A'-C') and the quantification of the Vg patterns in a rectangular region defined as in Fig. 1A in AU (A"'-C') and RU (A"'-C',') units as in Fig. 1. The quantifications of the Vg patterns in A"'-C'”, are normalized to DAPI levels as in Sup. Fig. 1. Labels in panels B-B"' and C-C"', are as in A-A'”.(D) Normalized Vg profiles of all the discs color-coded according to their DV length. (E) Relative width of the Vg gradient (as defined in Fig. 1G) as a function of DV length; each dot in the graph corresponds to a different disc and is color-coded as in D. The solid line shows the linear regression of the data and the Pearson Correlation Coefficient, $\mathrm{r}$, of the regression is displayed. The p-value corresponds to a Student-t statistical test assuming a zero-slope of the regression line as a null hypothesis. (F) Relative Vg intensity at the ventral (10\% of DV axis, top graph) and dorsal ( $90 \%$ of DV axis, bottom graph) tails. Each dot in the graphs corresponds to a different disc and is color-coded as in D. Linear regression and statistical analysis is performed as in E. 
A
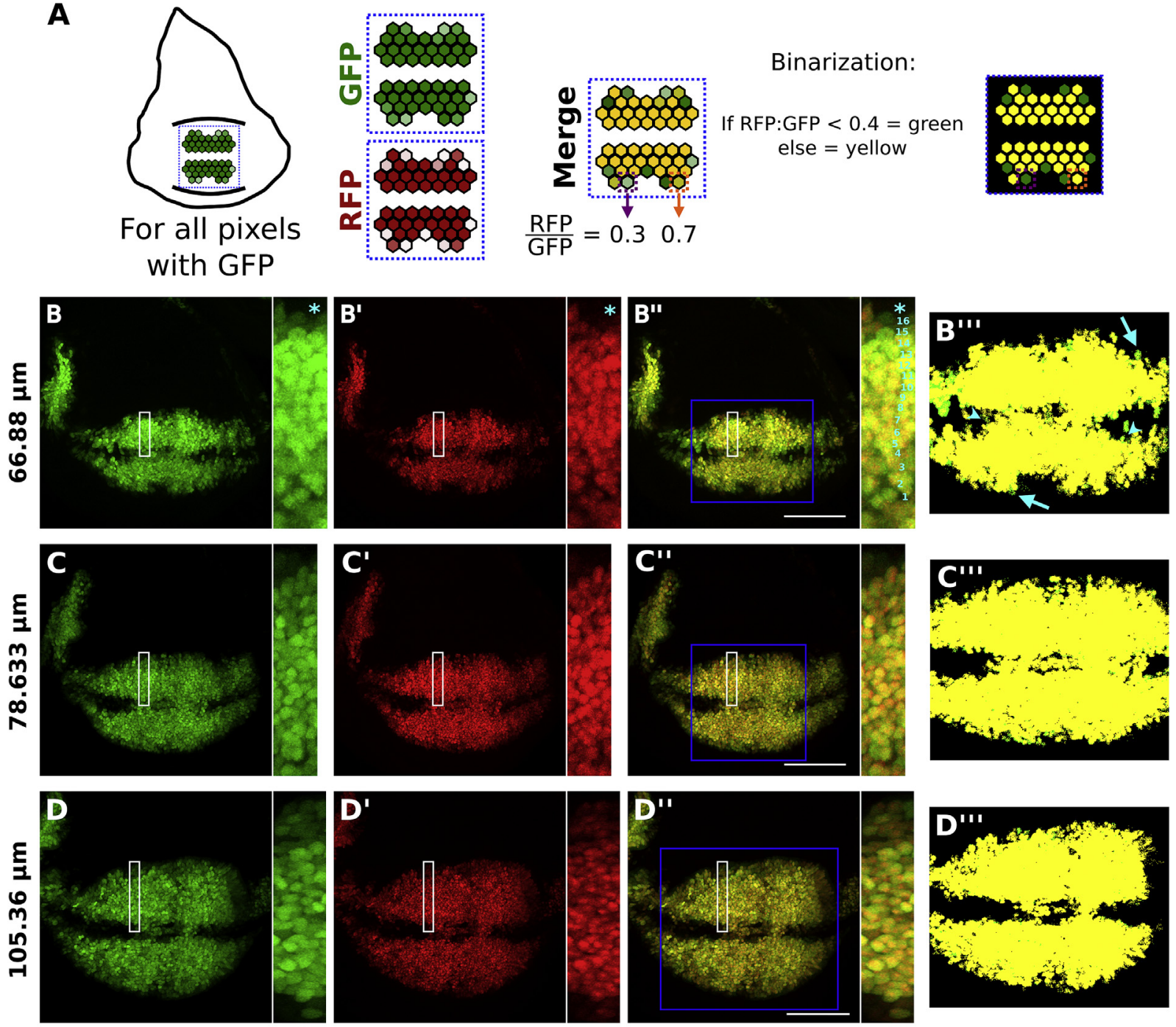

$\mathbf{E}$

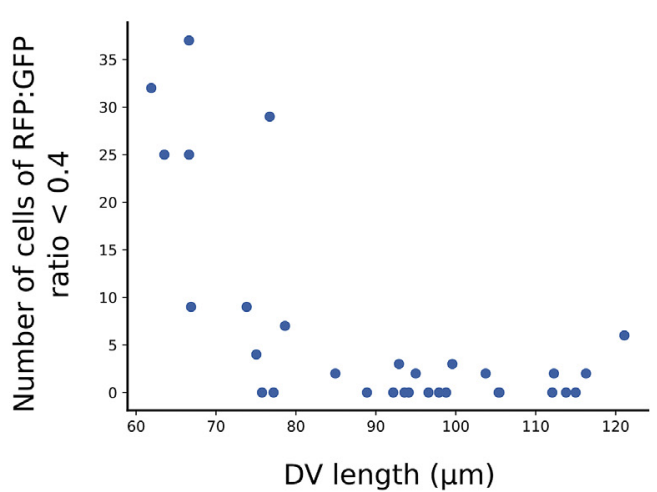

Fig. 4. $v g^{Q E}$ is activated in new cells located beyond the range of Wg signaling during early-to-mid third instar. Fixed wing discs from $v{ }^{Q E}{ }^{Q E a l 4}$, UASTransTimer larvae (see Material and Methods). (A) Scheme of the quantification approach. Only GFP-positive pixels within the wing pouch were considered in the analysis (see Material and Methods). For each of these pixels, we looked at the corresponding value in the RFP channel and computed the RFP to GFP ratio; ratio values below 0.4 (see Materials and Methods) were considered as pixels coming from cells that recently activated the $v g^{Q E}$. (B-D) Representative discs of different DV lengths that correspond to different developing times (distance between folds is displayed for each disc), that express RFP (B-D), GFP (B'-D') or both (B''-D'). A magnification within the rectangle is displayed for each panel in B-D. In B-B'" cells are numbered from the DV border to indicate the location (in number of cells) of some GFP-positive, RFP-negative cells (referred as "green cells" in the text; last cell is pointed with a cyan asterisk). The pixels that have a RFP to GFP below the 0.4 threshold are depicted in green, whereas pixels that have a RFP to GFP above the 0.4 threshold are depicted in yellow in B"'-D'”. In B"', cyan arrows and arrowheads point to green cells located in the periphery of the $v g^{Q E}$ pattern. (E) Quantification of the number of green cells (see Material and Methods) in each individual discs plotted vs. DV length. 


\subsection{Impairment of cell recruitment results in smaller, but well-proportioned} adult wings

We then asked whether cell recruitment has an effect on adult wing size. Therefore, we compared $d s>v g^{R N A i}$ vs. control adult wings (Fig. 5). Most adult wings of $d s>v g^{R N A i}$ animals show the normal vein patterns (Fig. 5A and B) but they are smaller than control wings (Fig. 5C). We found that this reduction in wing size is approximately uniform along the proximal-distal axis, as representative areas of both proximal (Fig. 5D, inset) and distal (Fig. 5E, inset) regions of the adult wing are similarly reduced in $d s>v g^{R N A i}$ animals (17 and $21 \%$, respectively; Fig. 5D-E). We also noticed a reduction of the hinge in these mutants (Fig. 5A-B), suggesting that the inhibition of $\mathrm{Vg}$ expression in the $d s$ domain may also have a non-cell-autonomous effect on hinge size, as has been previously proposed (Klein and Martinez-Arias, 1998; Liu et al., 2000). Since, the hinge is important for wing elongation during metamorphosis (Etournay et al., 2015; Casares and Mann, 2000), we considered if the reduction of wing size in $d s>v g^{R N A \mathrm{i}}$ could be an indirect effect of hinge size. If this were the case, the reduction would affect only the proximal-distal axis of the adult wing. However, we found that $d s>v g^{R N A i}$ wings maintain their proximal-distal (longitudinal) to anterior-posterior (transversal) proportions, as the ratio of longitudinal to transversal dimensions is not statistically different between control and mutant adult wings (Fig. 5F). Therefore, we conclude that the impairment of cell recruitment in $d s>$ $v g^{R N A i}$ animals significantly affects adult wing size but not its proportions.

\subsection{Without cell proliferation, cell recruitment drives some growth but} gives rise to very small wings

The previous experiment shows that in the absence of cell recruitment wings are smaller, suggesting that cell recruitment does contribute to adult wing size. For completeness, we also did the opposite experiment, namely, we asked how do wings turn out when cell recruitment is present, but cell proliferation is inhibited by the expression of an interference RNA of the cell-cycle gene string (stg) in the pattern of the $v g^{Q E}\left(v g^{Q E}\right.$ $>s t g^{R N A i}$ ). Note that in this experiment, cells outside of the $v g^{Q E}$ domain, but within the wing pouch, can proliferate and expand the population of "recruitable" cells. As expected, $v g^{Q E}>s g^{R N A i}$ discs have a clear reduction in cell proliferation with respect to control discs in the $v g^{Q E}$ domain (Fig. 6A and B). Despite this reduction in cell proliferation, cells do not

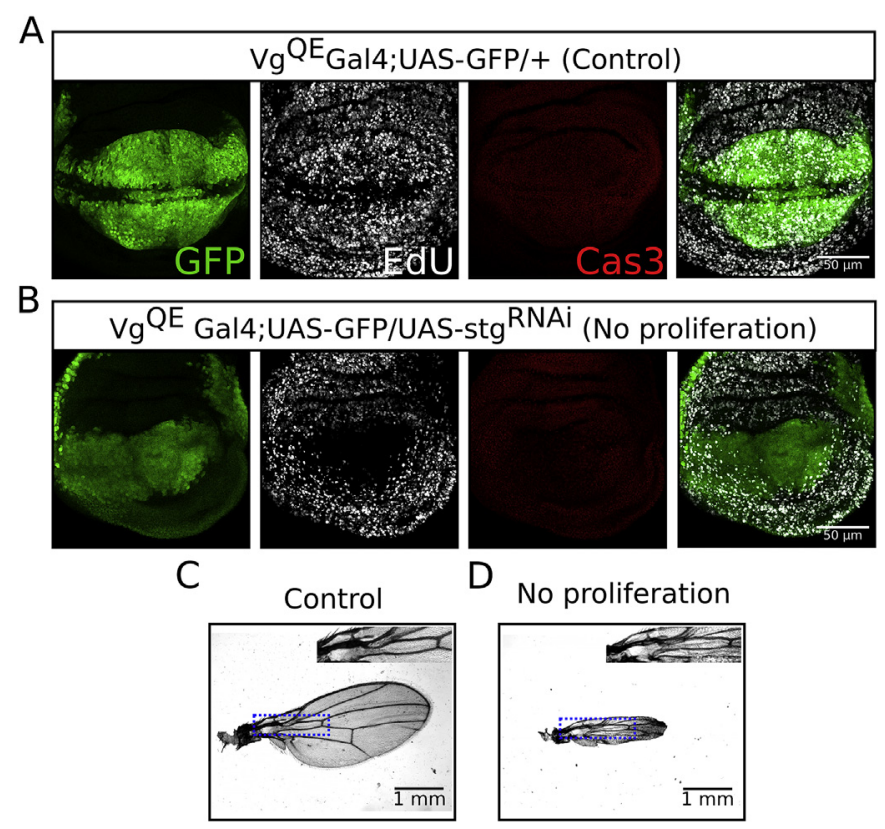

Fig. 6. Without cell proliferation, recruitment contributes to growth but cannot rescue adult wing size. A UAS-stg ${ }^{R N A i}$ stock (that blocks the cell-cycle progression) was crossed to a $v g^{Q E}$ Gal4; UAS-GFP stock to impair cell proliferation in the $v g^{Q E}$ region $\left(v g^{Q E}>s t g^{R N A i}\right.$ ). (A and B) Immunofluorescence images of control (A) and $v g^{Q E}>s g^{R N A i}$ (B) wing discs. From left to right are depicted: the $v g^{Q E}$ expression region (marked with GFP, green), EdU staining (white), Cas3 staining (red), and the merged images (GFP and EdU only). (C and D) Representative adult wings from the control (C) and $v g^{Q E}>s g^{R N A i}$ (D) animals. Inset shows a magnification of the proximal area marked by the blue-dotted rectangle.

seem to be outcompeted by their neighbors, as apoptosis remains negligible in these discs (Fig. 6A and B). When comparing the adult phenotypes, we found that wings of $v g^{Q E}>s t g^{R N A i}$ animals are notably smaller than their corresponding controls (Fig. 6C and D), indicating that cell recruitment on its own cannot rescue wing size in the absence of cell proliferation in $v g^{Q E}$ cells. However, the size and pattern of the proximalmost region of the wing is strikingly maintained in $v g^{Q E}>s g^{R N A i}$ animals
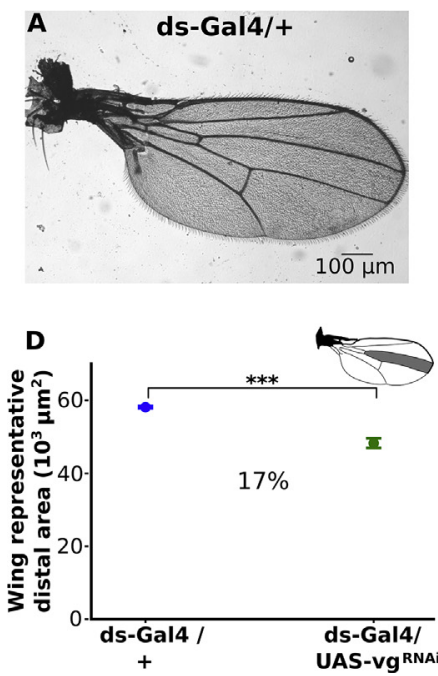
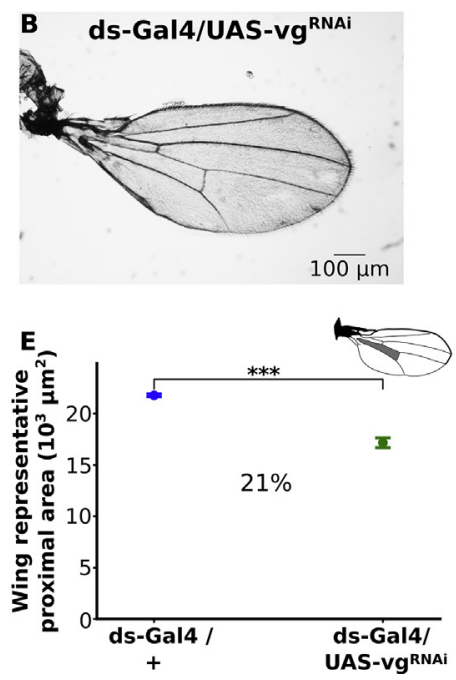
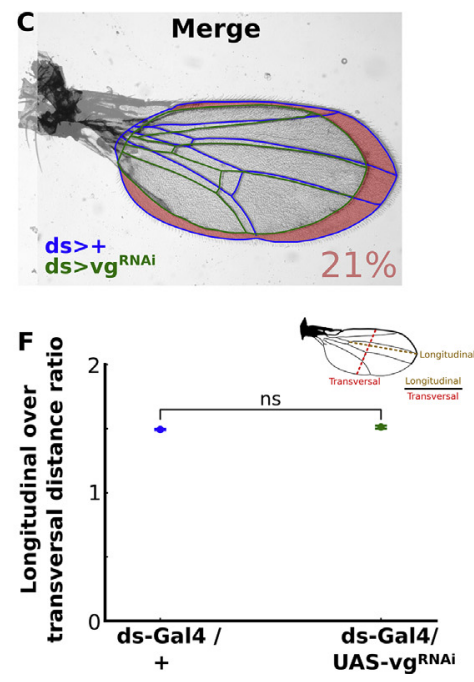

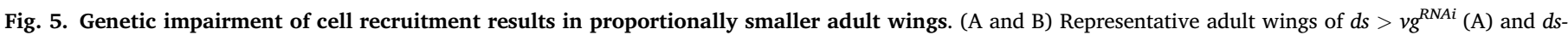

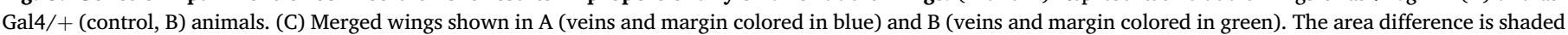

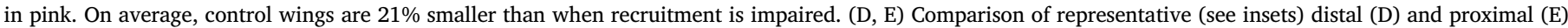

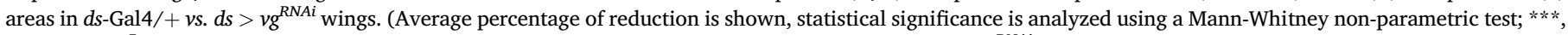

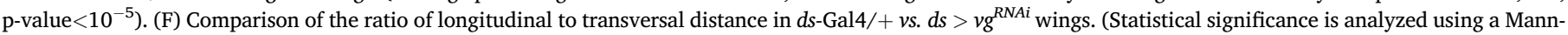
Whitney non-parametric test; ns, p-value $>0.01$ ). 
(Fig. 6C and D insets). These data confirm that cell proliferation is the major driver of wing growth and cell recruitment alone has a smaller contribution to wing size. We conclude that cell proliferation and cell recruitment additively contribute to adult wing size.

\section{Discussion}

How gene expression patterns that determine cell fate are established and contribute to organ size are fundamental questions in developmental biology. There are two predominant models by which gene expression patterns are coordinated with organ growth. In the first model, patterns continuously depend on morphogen signaling; when the morphogen gradient changes, the patterns also change accordingly (Gurdon et al., 1995). In particular, if the morphogen scales to organ size, the patterns that depend on the morphogen would maintain their proportions relative to the final size (Averbukh et al., 2014). In the second model, a morphogen gradient establishes pre-patterns of gene expression that are then locked by positive feedback regulation (Nahmad and Lander, 2011). Once cells are locked in a determined fate, the final patterns are no longer dependent on the morphogen but are determined by organ growth. Particularly, when growth is uniform, this results in patterns that scale with size throughout development. In both of these models, the patterning process adjusts to organ growth and the final patterns are invariant to organ size. Our data on patterning of the wing selector gene, $v g$, in the Drosophila wing disc support an additional conceptual model. After being established by Notch, Wg, and Dpp signaling, Vg expression is maintained through cell divisions by a mechanism involving Polycomb/Trithorax Responsive Elements and positive feedback on itself. In a tissue that grows uniformly like the wing disc, this would result in a pattern that scales with tissue size. However, we find that the Vg gradient overscales relative to DV axis length (Fig. 1). This cannot be explained by non-uniform cell proliferation, apoptosis, cell size, or a change in pattern of oriented cell divisions (Sup. Figs. 5, 14, 15). Instead, our modeling results suggest that $\mathrm{Vg}$ overscaling could be explained by a cell recruitment mechanism (Fig. 2). Experimentally, when cell recruitment is genetically blocked, the overscaling phenotype is mostly lost (Fig. 3). We provide direct evidence of the cell recruitment dynamics during $\mathrm{Vg}$ patterning (Fig. 4) and show that cell recruitment contributes to about $20 \%$ of adult wing size (Fig. 5). Finally, we show that when cell proliferation is blocked, cell recruitment cannot rescue wing size, suggesting that cell proliferation and cell recruitment additively contribute to organ growth in this system (Fig. 6).

Based on qualitative evidence on $\mathrm{Vg}$ expansion in genetic mosaics, Zecca and Struhl identified the molecular mechanisms of recruitment and proposed that Vg-dependent cell recruitment could contribute to growth of the Drosophila wing disc (Zecca and Struhl, 2007a, 2010). However, it remained unclear when and to what extent cell recruitment actually contributes to normal patterning and growth of the disc. Our study shows that cell recruitment works by overexpanding the endogenous pattern of a selector gene, thus contributing to both patterning and organ growth. To our knowledge, this is the first study where cell recruitment is directly demonstrated during wild-type development and where its contributions to pattern formation and organ growth are quantitatively determined.

$\mathrm{Wg}$ signaling is absolutely required for $\mathrm{Vg}$ expression and propagation along the DV axis (Zecca and Struhl, 2007a). But is Wg signaling the determining factor of the overscaling dynamics of the $\mathrm{Vg}$ pattern? By overexpressing the $\mathrm{Wg}$ receptor, Frizzled2 in individual cells, a recent study has shown that the maximum range of $\mathrm{Wg}$ signaling is 11 cells from the DV boundary (Chaudhary et al., 2019). We determined the position of cells that acquire new expression of the GFP reporter in the TransTimer system and show that some of these cells are located up to 16 cells away from the DV boundary (Fig. 4B-B"' asterisk). How can we conciliate the requirement of $\mathrm{Wg}$ signaling for cell recruitment with the observation that cells are being recruited outside the reported range of Wg signaling? We think that $v g$ expression may depend on memory of earlier, pouch-wide Wg signaling activity (Alexandre et al., 2014). If this were indeed the case, $\mathrm{Wg}$ signaling would only be a permissive factor and would not play a leading role in establishing the range or overscaling of Vg patterning.

In this study, we focused on Vg patterning along the DV axis, but Dpp signaling organizes patterning of the disc along the anterior-posterior (AP) axis and Dpp is also an input signal for $v g$ expression (Kim et al., 1996). Importantly, our analysis using the TransTimer system shows new activation of the GFP reporter in cells located in the anterior and posterior ends of the disc (see Fig. 4B"'). Therefore, how Dpp signaling contributes to cell recruitment along the AP axis and participates in the 2-dimensional $\mathrm{Vg}$ pattern is a promising perspective left to future work.

Our experiments show that new cells are being incorporated into the $v g^{Q E}$ pattern, i.e. new cells get recruited, and reveal that this recruitment process is temporally regulated (Fig. 4). This correlates with the overscaling of the Vg pattern that spans over discs of similar sizes (Fig. 1F-H). We propose that cell recruitment is a continuous process that is initiated by the transcriptional activation of $v g$ in the early-mid stage of the third larval instar, and continues until the end of the third instar by increasing the levels of $\mathrm{Vg}$ until they reach an appropriate level for wing-fate differentiation. At the molecular level, the initiation of the recruitment process is likely driven by the Ft-Ds polarization signal that translocate Yki to the nucleus of the newly-recruited cell and results in increasing $v g$ levels. The fact that this increase in Vg expression is slow and spans over half of the third instar (approximately $24 \mathrm{~h}$ ) suggests that the rates of $\mathrm{Vg}$ upregulation are position-dependent, possibly dictated by the strength of the Ft-Ds polarization which decays from its source near the DV boundary towards ventral- and dorsal-most positions (Wortman et al., 2017). This model of cell recruitment suggests that the spatial range of the process is limited by the length-scale of the Ft-Ds polarization signal and by the availability of "recruitable" cells within the wing pouch.

We show that cell recruitment contributes to approximately one fifth of the total adult wing size (Fig. $5 \mathrm{C}$ ). An interesting question that remains is to understand the purpose of cell recruitment as a growth mechanism. In other words, what is the advantage of this developmental design over simply adjusting cell sizes or cell proliferation rates to achieve a target final size? It is possible that cell recruitment plays a role in conferring some sort of robustness to developmental growth control, by providing a fine-tuning contribution to growth. While cell proliferation in the wing disc doubles the number of wing cells approximately every 8-10 h (Mao et al., 2013), cell recruitment can incorporate $30-50$ additional cells into the wing primordium between early and mid third instar development (Fig. 4E). Thus, cell recruitment may serve as a mechanism to provide additional growth at a slower rate than cell proliferation.

The fact that we found that cell recruitment and cell proliferation cannot rescue growth in the absence of each other (Figs. 5 and 6) does not necessarily mean that these processes are completely independent. For example, cell recruitment may buffer small variations in cell proliferation and may participate in growth control to attain wings of the right size (Day and Lawrence, 2000). Thus, the interplay between cell proliferation and cell recruitment under moderate perturbations remains to be investigated in more detail.

Recent studies have showed that a cell recruitment mechanism is present in early thyroid and kidney development in mammals (Fagman et al., 2006; Lindström et al., 2018), but it is unclear how much recruitment contributes to organ growth or what are the molecular players of the recruitment signal in these contexts. Given the widespread conservation of the recruitment signal components that operate in the Drosophila wing, it would be interesting to explore whether the homologous cell recruitment signal operates as a growth-by patterning mechanism in other developing organs.

\section{Material and Methods}

\subsection{Fly stocks and genetics}

The following stocks of Drosophila melanogaster were used: $y, w$ 
provided by Fanis Missirlis (Cinvestav, Mexico); UAS-TransTimer provided by Li He (Norbert Perrimon's Lab, Harvard Medical School, USA); dppGal4 (Bloomington Drosophila Stock Center, BDSC \#1553); Act > STOP > LacZ (BDSC \#6355); UAS-hid (BDSC \#65403); UAS- $v g^{R N A i}$ (Vienna Drosophila Resource Center \# 16896); $d s$-Gal4, UAS-GFP (II) provided by Gary Struhl (Columbia University, New York, USA); $v g^{Q E_{-}}$ Gal4 (III) (BDSC \#8229); UAS-stg ${ }^{R N A i}$ (obtained from Arthur Lander, University of California - Irvine, USA). Unless indicated otherwise, flies were kept at $25{ }^{\circ} \mathrm{C}$ during crossing and during development. The $y, w$ stock was used as wild-type flies (Fig. 1). For recruitment-impairment experiments (Figs. 3 and 5), dsGal4, UAS-GFP flies were crossed to UAS- $v g^{R N A i}$ (for recruitment inhibition) or the $y, w$ stock (control) and were kept at $25{ }^{\circ} \mathrm{C}$ during egg laid $(24 \mathrm{~h})$ and then changed to $29{ }^{\circ} \mathrm{C}$ during development to increase Gal4 system efficiency. For the evaluation of the $v{ }^{Q E}$ dynamics (Fig. 4), UAS-TransTimer reporter, a destabilized GFP (dGFP) protein combined with a stable RFP (He et al., 2019), was placed under $v g^{Q E}$ Gal 4 driver to quantify cells that recently activated this enhancer. To impair cell proliferation in the vg normal expression pattern (Fig. 6), $v g^{Q E}$ Gal4; UAS-GFP flies are crossed to UAS-stg ${ }^{R N A i}$ flies or the $y, w$ stock (control) at $29^{\circ} \mathrm{C}$ to increase Gal4 system efficiency.

\subsection{Immunostaining}

Wing imaginal discs were dissected from third-instar larvae of both sexes. For larva dissected at a specific age AEL (Fig. 1), embryos were collected in apple juice agar plates for $4 \mathrm{~h}$ and then they were transferred to fly food bottles for the indicated times (84, 92, 100 and $108 \mathrm{~h} \mathrm{AEL,}$ respectively). After dissection in a stereoscopic microscope (Nikon SMZ800), discs were fixed in PEM-T (PEM with $0.1 \%$ of Triton X-100) with 4\% paraformaldehyde, washed 3 times and blocked in PEM-T with $0.5 \%$ of BSA (Bovine Serum Albumin) for $2 \mathrm{~h}$ at room temperature. Then, samples were stained with primary antibodies at $4{ }^{\circ} \mathrm{C}$ overnight at the following dilutions: rabbit anti- $\mathrm{Vg}$ (a gift from Sean Carroll and Kristen Guss, 1:200), mouse anti-Disc large (1:50, Hybridoma Bank, ID: AB 528203, 4F3 anti-discs large was deposited to the DSHB by Goodman, C. [DSHB Hybridoma Product 4F3 anti-discs large]), rabbit anti-Caspase 3 (Cell Signaling Technology, Cat\# 9661, 1:200), mouse anti-beta Galactosidase (Promega, Cat \#Z378A. 1:1000). DAPI (1:1000) was used to stain nuclei. 5-ethynyl-2'-deoxyuridine (EdU) labeling was performed using the Click-iT EdU Alexa Fluor 647 Imaging Kit (Invitrogen Cat\#C10340) following manufacturer instructions. Primary antibodies were detected with Alexa Fluor 488 anti-mouse and Alexa Fluor 647 antirabbit (1:1000). Imaging was done with a confocal microscope (Leica TCS SP8 Confocal Microscope) using a 63X oil-immersion objective.

\subsection{Image analysis and quantification}

The details about the quantification of $\mathrm{Vg}$ (Figs. 1 and 3) are provided in Sup. Fig. 1. All the quantifications were performed using Python 3 programming language (https://www.python.org/download/release s/3.0/). The Python packages used for quantification and statistics were NumPy (http://www.numpy.org/), pandas (https://pandas.py data.org/), and SciPy (https://www.scipy.org/).

For TransTimer reporter quantification (Fig. 4), we selected all the GFP pixels with intensity values above a detecting threshold in the wing pouch for each disc; this threshold was set by ranking all the pixels within by their GFP intensity in three different groups using the machine learning K-Means function from scikit-learn python package (https://sci kit-learn.org/stable/index.html) and all the pixels in the lower group were taken away. Next, we looked for the corresponding RFP channel and computed a ratio of RFP over GFP intensities. If this ratio was lower than 0.4 at a specific pixel (this value corresponds to the ratio at the half maximum for GFP taken from Fig. 3b in He et al., 2019), we marked this pixel in green and considered it as new activation of the $v g^{Q E}$, the ones above the threshold were marked in yellow (Fig. 4A). To count green cells (recruited cells), we used the Analyze Particles tool in ImageJ (https://imagej.net/) on the green channel only of the thresholded images (e.g. Fig. 4B"')' We set the parameters of the Analyze Particles that fitted best the manual cell counting of two independent observers on three different images.

To quantify cell areas in wing discs (Sup. Fig. 15), third instar wing discs were immunostained with a Discs Large (Dlg) antibody that labels cell membranes. Images were binarized in ImageJ using the Threshold tool and then the Watershed tool. The holes between cells were eliminated to allow the measurement of the area of each individual cell using the Analyze Particles tool, to avoid counting individual or a few pixels together as cells, we set a minimum threshold to consider a cell.

\subsection{Wing mounting and area quantification}

Adult flies were dehydrated overnight in $70 \%$ ethanol and then separated by gender. Wings were dissected in 50\% ethanol. The isolated wings were mounted and dried in a plate at $60^{\circ} \mathrm{C}$. Imaging of adult wings was done using a bright-field microscope (Nikon eclipse Ci-L/Ci-S) using a 4X objective. Wings were dissected from female and male flies and were independently analyzed. Wing distances and areas were quantified using the straight line and polygon selection tools in the ImageJ software.

\subsection{Simulations}

All simulations were performed using Morpheus 1.9.3 software (http s://imc.zih.tu-dresden.de/wiki/morpheus). The time dependence of the $\mathrm{Vg}$ concentration was modeled by means of an ordinary differential equation for each cell (Fig. 2A), and the tissue was modeled by a Cellular Pots Model (CPM). Examples of the. xml files used to perform simulations are provided as supplementary files.

\subsection{Image processing and data visualization}

All the images were processed and analyzed using ImageJ/Fiji software, the matplotlib (https://matplotlib.org/), pandas (https:// pandas.pydata.org/), and NumPy (http://www.numpy.org/) python packages. The data, after being analyzed, were visualized with the seaborn (https://seaborn.pydata.org/) and Matplotlib python packages.

\section{Funding}

This work was supported by the Consejo Nacional de Ciencia y Tecnología of Mexico (Conacyt), [grant number CB-2014-01-236685] to M. Nahmad. and by the grants from Agencia Nacional de Promoción Científica y Tecnológica of Argentina, [grant numbers PICT-2014-3469, PICT2017-2307] to O. Chara. L. M. Muñoz-Nava and M. Flores-Flores received financial support from Conacyt's graduate scholarship program. O. Chara is a career researcher from Consejo Nacional de Investigaciones Científicas y Técnicas of Argentina.

\section{Declaration of competing interest}

No competing interests declared.

\section{Acknowledgments}

We thank Li He from Norbert Perrimon's Lab for kindly providing us the UAS-TransTimer flies and Fanis Missirlis for sharing flies and giving us critical comments on the manuscript on its early stages. We also thank Jose Luis Fernandez for technical assistance, and members of the Nahmad and Chara laboratories for interesting discussions.

\section{Appendix A. Supplementary data}

Supplementary data to this article can be found online at https:// doi.org/10.1016/j.ydbio.2020.03.009. 


\section{References}

Ahmad, K., Spens, A.E., 2019. Separate Polycomb Response Elements control chromatin state and activation of the vestigial gene. PLoS Genet. 15, 1-21. https://doi.org/ 10.1371/journal.pgen.1007877.

Alexandre, C., Baena-Lopez, A., Vincent, J.P., 2014. Patterning and growth control by membrane-tethered wingless. Nature 505, 180-185. https://doi.org/10.1038/ nature12879.

Averbukh, I., Ben-Zvi, D., Mishra, S., Barkai, N., 2014. Scaling morphogen gradients during tissue growth by a cell division rule. Development 141, 2150-2156. https:// doi.org/10.1242/dev.107011.

Baena-Lopez, L.A., 2003. Genetic requirements of vestigial in the regulation of Drosophila wing development. Development 130, 197-208. https://doi.org/10.1242/ dev.00187.

Baena-López, L.A., Baonza, A., García-Bellido, A., 2005. The orientation of cell divisions determines the shape of Drosophila organs. Curr. Biol. 15, 1640-1644. https:// doi.org/10.1016/j.cub.2005.07.062.

Baena-López, L.A., García-Bellido, A., 2006. Control of growth and positional information by the graded vestigial expression pattern in the wing of drosophila melanogaster. Proc. Natl. Acad. Sci. U.S.A. 103, 13734-13739. https://doi.org/10.1073/ pnas.0606092103.

Beira, J.V., Paro, R., 2016. The legacy of Drosophila imaginal discs. Chromosoma 125, 573-592. https://doi.org/10.1007/s00412-016-0595-4.

Brand, A.H., Perrimon, N., 1993. Targeted gene expression as a means of altering cell fates and generating dominant phenotypes. Development 118, 401-415. https:// doi.org/10.1101/lm.1331809.

Bryant, S.V., Gardiner, D.M., 2016. The relationship between growth and pattern formation. Regeneration 3, 103-122. https://doi.org/10.1002/reg2.55.

Casares, F., Mann, R.S., 2000. A dual role for homothorax in inhibiting wing blade development and specifying proximal wing identities in Drosophila. Development 127, 1499-1508.

Chaudhary, V., Hingole, S., Frei, J., Port, F., Strutt, D., Boutros, M., 2019. Robust Wnt signaling is maintained by a $\mathrm{Wg}$ protein gradient and $\mathrm{Fz} 2$ receptor activity in the developing Drosophila wing. Development 146. https://doi.org/10.1242/ dev.174789 dev174789.

Day, S.J., Lawrence, P.A., 2000. Measuring dimensions: the regulation of size and shape Development 127, 2977-2987.

Dekanty, A., Milán, M., 2011. The interplay between morphogens and tissue growth EMBO Rep. 12, 1003-1010. https://doi.org/10.1038/embor.2011.172.

Etournay, R., Popović, M., Merkel, M., Nandi, A., Blasse, C., Aigouy, B., Brandl, H., Myers, G., Salbreux, G., Jülicher, F., Eaton, S., 2015. Interplay of cell dynamics and epithelial tension during morphogenesis of the Drosophila pupal wing. Elife 4, 1-51. https://doi.org/10.7554/eLife.07090.

Fagman, H., Andersson, L., Nilsson, M., 2006. The developing mouse thyroid: embryonic vessel contacts and parenchymal growth pattern during specification, budding, migration, and lobulation. Dev. Dynam. 235, 444-455. https://doi.org/10.1002/ dvdy.20653.

Glazier, J.A., Graner, F., 1993. Simulation of the differential adhesion driven rearrangement of biological cells. Phys. Rev. E 47, 2128-2154. https://doi.org/ 10.1103/PhysRevE.47.2128.

González-Gaitán, M., Capdevila, M.P., García-Bellido, A., 1994. Cell proliferation patterns in the wing imaginal disc of Drosophila. Mech. Dev. 46, 183-200. https://doi.org/ 10.1016/0925-4773(94)90070-1.

Gou, J., Stotsky, J.A., Othmer, H.G., 2020. Growth control in the Drosophila wing disk. WIREs Syst. Biol. Med. 1-28. https://doi.org/10.1002/wsbm.1478.

Graner, F., Glazier, J.A., 1992. Simulation of biological cell sorting using a twodimensional extended Potts model. Phys. Rev. Lett. 69, 2013-2016. https://doi.org/ 10.1103/PhysRevLett.69.2013.

Gurdon, J.B., Mitchell, A., Mahony, D., 1995. Direct and continuous assessment by cells of their position in a morphogen gradient. Nature 376, 520-521.

Halder, G., Polaczyk, P., Kraus, M.E., Hudson, A., Kim, J., Laughon, A., Carroll, S., 1998. The Vestigial and Scalloped proteins act together to directly regulate wing-specific gene expression in Drosophila. Genes Dev. 12, 3900-3909. https://doi.org/10.1101/ gad.12.24.3900

Hariharan, I.K., Bilder, D., 2006. Regulation of imaginal disc growth by tumor-suppressor genes in Drosophila. Annu. Rev. Genet. 40, 335-361. https://doi.org/10.1146/ annurev.genet.39.073003.100738.

He, L., Binari, R., Huang, J., Falo-Sanjuan, J., Perrimon, N., 2019. In vivo study of gene expression with an enhanced dual-color fluorescent transcriptional timer. Elife 8 , 1-20. https://doi.org/10.7554/elife.46181.

Heberlein, U., Singh, C.M., Luk, A.Y., Donohoe, T.J., 1995. Growth and differentiation in the Drosophila eye coordinated by hedgehog. Nature 373, 709-711. https://doi.org/ 10.1038/373709a0.

Irvine, K.D., Vogt, T.F., 1997. Dorsal-ventral signaling in limb development. Curr. Opin. Cell Biol. 9, 867-876.
Kim, J., Sebring, A., Esch, J.J., Kraus, M.E., Vorwerk, K., Magee, J., Carroll, S.B., 1996. Integration of positional signals and regulation of wing formation and identity by Drosophila vestigial gene. Nature 382, 133-138. https://doi.org/10.1038/382133a0.

Klein, T., Arias, A.M., 1998. Different spatial and temporal interactions between Notch, wingless, and vestigial specify proximal and distal pattern elements of the wing in Drosophila. Dev. Biol. 194, 196-212. https://doi.org/10.1006/dbio.1997.8829.

Klein, T., Arias, A.M., 1999. The Vestigial gene product provides a molecular context for the interpretation of signals during the development of the wing in Drosophila. Development 126, 913-925.

Lander, A.D., 2011. Pattern, growth, and control. Cell 144, 955-969. https://doi.org/ 10.1016/j.cell.2011.03.009.

Lindström, N.O., De Sena Brandine, G., Tran, T., Ransick, A., Suh, G., Guo, J., Kim, A.D., Parvez, R.K., Ruffins, S.W., Rutledge, E.A., Thornton, M.E., Grubbs, B., McMahon, J.A., Smith, A.D., McMahon, A.P., 2018. Progressive recruitment of mesenchymal progenitors reveals a time-dependent process of cell fate acquisition in mouse and human nephrogenesis. Dev. Cell 45, 651-660. https://doi.org/10.1016/ j.devcel.2018.05.010 e4.

Liu, X., Grammont, M., Irvine, K.D., 2000. Roles for Scalloped and Vestigial in regulating cell affinity and interactions between the wing blade and the wing hinge. Dev. Biol. 228, 287-303. https://doi.org/10.1006/dbio.2000.9939.

Mao, Y., Tournier, A.L., Hoppe, A., Kester, L., Thompson, B.J., Tapon, N., 2013. Differential proliferation rates generate patterns of mechanical tension that orient tissue growth. EMBO J. 32, 2790-2803. https://doi.org/10.1038/emboj.2013.197.

Milán, M., Campuzano, S., García-Bellido, A., 1997. Developmental parameters of cell death in the wing disc of Drosophila. Proc. Natl. Acad. Sci. U. S. A. 94, 5691-5696. https://doi.org/10.1073/pnas.94.11.5691.

Nahmad, M., Lander, A.D., 2011. Spatiotemporal mechanisms of morphogen gradient interpretation. Curr. Op. Gen. Dev. 21, 726-731. https://doi.org/10.1016/ j.gde.2011.10.002.

Pérez, L., Barrio, L., Cano, D., Fiuza, U.-M., Muzzopappa, M., Milán, M., 2011. EnhancerPRE communication contributes to the expansion of gene expression domains in proliferating primordia. Development 138, 3125-3134. https://doi.org/10.1242/ dev.065599.

Resino, J., Salama-Cohen, P., Garcia-Bellido, A., 2002. Determining the role of patterned cell proliferation in the shape and size of the Drosophila wing. Proc. Natl. Acad. Sci. U. S. A. 99, 7502-7507. https://doi.org/10.1073/pnas.072208199.

Schwank, G., Basler, K., 2010. Regulation of organ growth by morphogen gradients. Cold Spring Harb. Perspect. Biol. 2, a001669. https://doi.org/10.1101/ cshperspect.a001669.

Schwank, G., Tauriello, G., Yagi, R., Kranz, E., Koumoutsakos, P., Basler, K., 2011. Antagonistic growth regulation by Dpp and Fat drives uniform cell proliferation. Dev. Cell 20, 123-130. https://doi.org/10.1016/j.devcel.2010.11.007.

Strutt, D.I., Wiersdorff, V., Mlodzik, M., 1995. Regulation of furrow progression in the Drosophila eye by cAMP-dependent protein kinase A. Nature 373, 705-709. https:// doi.org/10.1038/373705a0.

Sui, L., Pflugfelder, G.O., Shen, J., 2012. The Dorsocross T-box transcription factors promote tissue morphogenesis in the Drosophila wing imaginal disc. Development 139, 2773-2782. https://doi.org/10.1242/dev.079384.

Vollmer, J., Casares, F., Iber, D., 2017. Growth and size control during development. Open Biol. 7, 170190. https://doi.org/10.1098/rsob.170190.

Wartlick, O., Mumcu, P., Kicheva, A., Bittig, T., Seum, C., Julicher, F., GonzalezGaitan, M., 2011. Dynamics of Dpp signaling and proliferation control. Science 331, 1154-1159. https://doi.org/10.1126/science.1200037.

Williams, J. a, Bell, J.B., Carroll, S.B., 1991. Control of Drosophila wing and haltere development by the nuclear vestigial gene product. Genes Dev. 5, 2481-2495. https://doi.org/10.1101/gad.5.12b.2481.

Williams, J. a, Paddock, S.W., Carroll, S.B., 1993. Pattern formation in a secondary field: a hierarchy of regulatory genes subdivides the developing Drosophila wing disc into discrete subregions. Development 117, 571-584.

Williams, J.A., Paddock, S.W., Vorwerk, K., Carroll, S.B., 1994. Organization of wing formation and induction of a wing-patterning gene at the dorsal/ventral compartment boundary. Nature 368, 299-305. https://doi.org/10.1038/368299a0.

Wortman, J.C., Nahmad, M., Zhang, P.C., Lander, A.D., Yu, C.C., 2017. Expanding signaling-molecule wavefront model of cell polarization in the Drosophila wing primordium. PLoS Comput. Biol. 13, e1005610. https://doi.org/10.1371/ journal.pcbi.1005610.

Yu, F.-X., Zhao, B., Guan, K.-L., 2015. Hippo pathway in organ size control, tissue homeostasis, and cancer. Cell 163, 811-828. https://doi.org/10.1016/ j.cell.2015.10.044.

Zecca, M., Struhl, G., 2007a. Recruitment of cells into the Drosophila wing primordium by a feed-forward circuit of vestigial autoregulation. Development 134, 3001-3010. https://doi.org/10.1242/dev.006411.

Zecca, M., Struhl, G., 2007b. Control of Drosophila wing growth by the vestigial quadrant enhancer. Development 134, 3011-3020. https://doi.org/10.1242/dev.006445.

Zecca, M., Struhl, G., 2010. A feed-forward circuit linking wingless, fat-dachsous signaling, and the warts-hippo pathway to Drosophila wing growth. PLoS Biol. 8, e1000386 https://doi.org/10.1371/journal.pbio.1000386. 